\title{
Improved in-vivo cardiac DTI using optimal b-values
}

\author{
Andrew D Scott ${ }^{1 *}$, Pedro Ferreira ${ }^{1,2}$, Sonia Nielles-Vallespin ${ }^{3,1}$, Laura-Ann McGill ${ }^{1,2}$, Philip J Kilner ${ }^{1,2}$, \\ Dudley J Pennell ${ }^{1,2}$, David Firmin ${ }^{1,2}$ \\ From 17th Annual SCMR Scientific Sessions \\ New Orleans, LA, USA. 16-19 January 2014
}

\section{Background}

There has been much recent interest in the microstructural information available using cardiac diffusion tensor imaging (cDTI)[1-3]. cDTI measures signal loss between a reference (b0) and a diffusion weighted image. The signal loss is caused by both diffusion and other sources of intravoxel incoherent motion, such as microvascular perfusion. By applying diffusion weighting to the reference image (bref), the microvascular perfusion component, which has a high apparent diffusion coefficient could be eliminated allowing a measurement of diffusion alone[4]. However, in order to provide a sufficient difference in signal intensity, the amount of diffusion encoding (b-value) must be higher than in previous cDTI studies. We compare mean diffusivity (MD) and fractional anisotropy (FA) derived from cDTI acquired with b-values between $b=50$ and $b=950 \mathrm{smm}^{-2}$ and separate diffusion from perfusion.

\section{Methods}

cDTI was performed in 10 healthy subjects ( 7 male, age 23-57, Siemens Skyra) using the stimulated echo single shot EPI with monopolar diffusion encoding sequence, described previously[1]. A single short axis slice in the mid-ventricle was imaged at $2.8 \times 2.8 \times 8 \mathrm{~mm}^{3}$ with 8 averages and 6 directions (+b0) at $\mathrm{b}=50,150,350,550$, $750,950 \mathrm{smm}^{-2}$. Pixel wise diffusion tensors were calculated using each b-value with $\mathrm{b} 0$ and also using all possible b-values as bref (e.g. $b=750$ vs. bref $=$ b0,50,150,350, $550 \mathrm{smm}^{-2}$ ). MD, FA and helical angle (HA) maps were derived in each case. For each subject the average diffusion weighted signal (averaged over all directions) at each b-value was calculated in the left ventricle.

\section{Results}

Figure 1A shows the signal loss in the left ventricle with b-value. A bi-exponential fit (fitted to $\mathrm{b}<1000 \mathrm{smm}^{-2}$ to avoid the noise floor), with diffusion $\left(D_{1}\right)$ and microvascular perfusion $\left(D_{2}\right)$ components matches the data more closely than the standard mono-exponential model $\left(R^{2}=0.995\right.$ vs. 0.986$)$. By $b=150 \mathrm{smm}^{-2}, D_{2}$ contributes approximately $1 \%$ of the signal. Figure $1 \mathrm{~B}$ gives mean $\mathrm{MD}$ and FA calculated using all pairs of b-values. With increasing bref the MD reduces and FA increases. MD is closest to $D_{1}$ with $b=750$ vs. bref $=150 \mathrm{smm}^{2}$. Figure 2 shows example cDTI parameter maps calculated using 4 pairs of $b$-values.

\section{Conclusions}

Increasing the b-value used in cDTI results in smoother MD and FA maps with less variation between subjects. Diffusion can be isolated from microvascular perfusion using a diffusion weighted reference images, which reduces the dependence of MD and FA on the b-value.

\section{Funding}

This work was performed at the NIHR funded Cardiovascular Biomedical Research Unit at The Royal Brompton Hospital. 

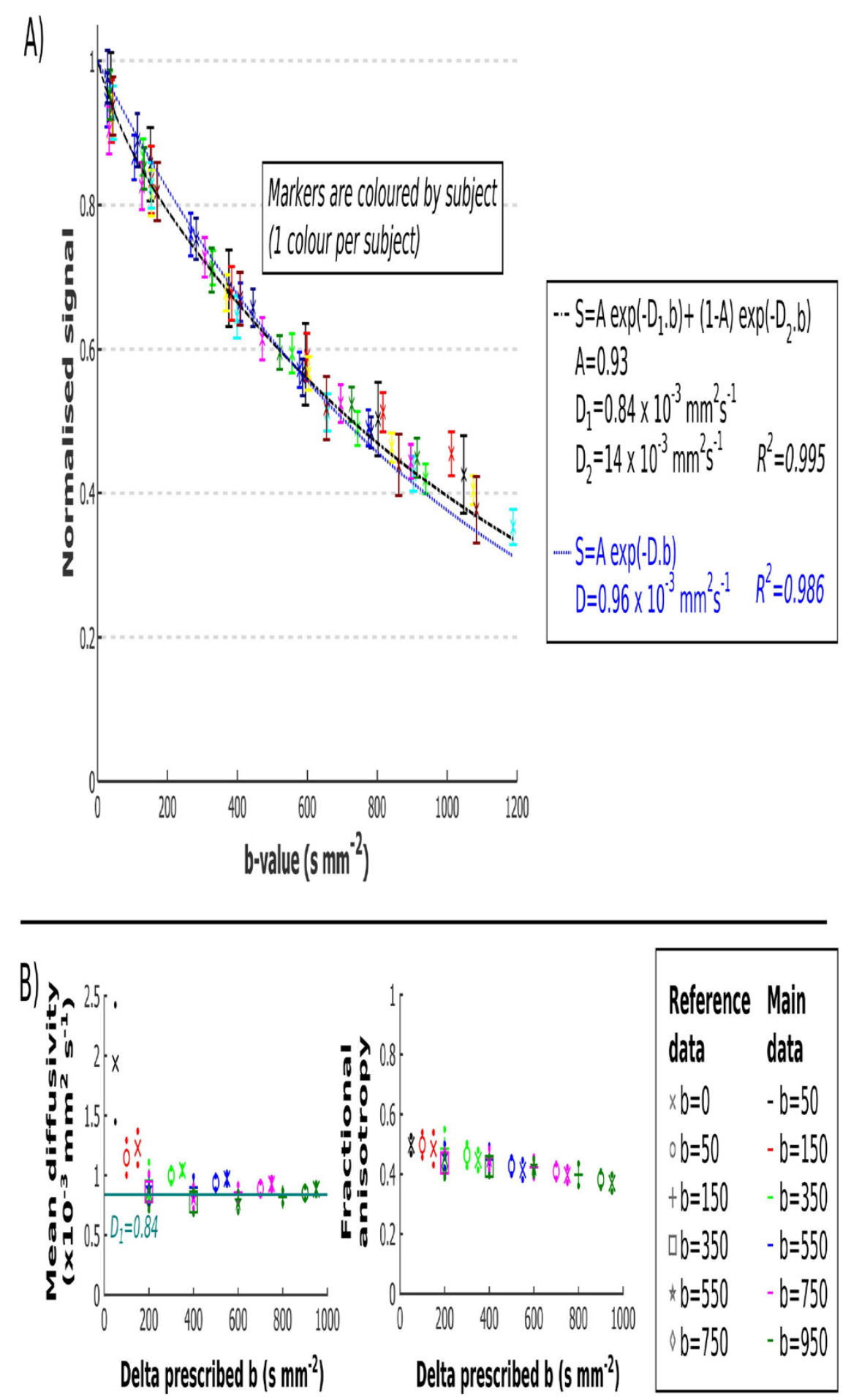

Figure 1 Normalised signal intensity (A) and derived CDTI parameters (B) plotted with b-value. Mono- and bi-exponential models are fitted to the normalised signal intensity vs. b-value corrected for heart rate (A). Mean ( \pm SD as small dots) MD and FA are plotted for every possible combination of b-value (marker colour) and bref (marker type) (B). 


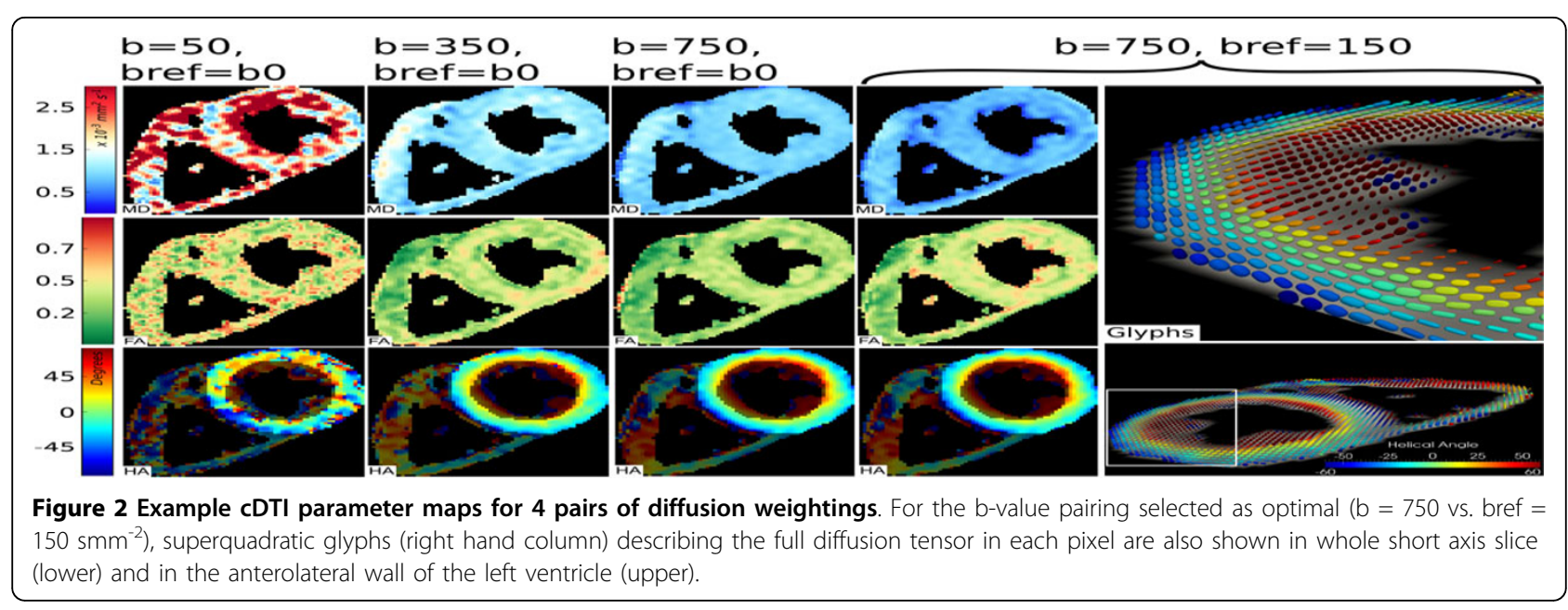

Authors' details

${ }^{1}$ Cardiovascular Biomedical Research Unit, The Royal Brompton Hospital, London, UK. ${ }^{2}$ National Heart and Lung Institute, Imperial College, London, UK. ${ }^{3}$ National Heart, Lung and Blood Institute, National Institute of Health, Bethesda, Maryland, USA.

Published: 16 January 2014

\section{References}

1. In MRM. Volume 70. Nielles-Vallespin; 2013:(2):454.

2. In JCMR. Volume 14. McGill; 2012:86.

3. Med Imag Anal Toussaint; 2013.

4. In Invest Radiol. Volume 47. Delattre; 2012:(11):662.

doi:10.1186/1532-429X-16-S1-027

Cite this article as: Scott et al.: Improved in-vivo cardiac DTI using

optimal b-values. Journal of Cardiovascular Magnetic Resonance 201416 (Suppl 1):O27.

Submit your next manuscript to BioMed Central and take full advantage of:

- Convenient online submission

- Thorough peer review

- No space constraints or color figure charges

- Immediate publication on acceptance

- Inclusion in PubMed, CAS, Scopus and Google Scholar

- Research which is freely available for redistribution 